\title{
Fast Inter Mode Decision Based on Hierarchical Homogeneous Detection and Cost Analysis for H.264/AVC Coders
}

\author{
Hung-Ming Wang, Ji-Kun Lin, and Jar-Ferr Yang \\ Department of Electrical Engineering \\ National Cheng Kung University \\ 1 University Road, Tainan, Taiwan 701, R.O.C.
}

\begin{abstract}
In H.264 advanced video coding (AVC), variable block size motion estimation plays an important role in compression of interframes. In this paper, we propose a fast inter prediction algorithm based on hierarchical homogeneous detection and cost analysis to select the best mode effectively. For each macroblock, we first detect that whether the macroblock is spatial homogeneous or not. For the non spatial homogeneous macroblock, we then perform the $16 \times 16$ motion estimation and examine if the $16 \times 16$ block is temporal homogeneous or not. Once the homogeneous macroblock is detected in the above process, the best mode will be chosen as 16x16 mode. For the non-homogeneous macroblock, we then execute $8 \times 8$ motion estimation and analyze the cost of $8 \times 8$ mode and $16 \times 16$ mode for deciding the best inter mode should be $16 \times 16$ mode or any other mode. The process for searching the best $8 \times 8$ block subtype is similar to the process for macroblocks. Finally, the best inter mode is decided by selecting the inter mode with least cost from the candidate modes. Experimental results show that our proposed algorithm can save about $32 \sim 54 \%$ computation time without introducing any noticeable performance degradation.
\end{abstract}

\section{INTRODUCTION}

In the digital epoch, people demand more and more high-quality multimedia services in their digital lives. Nevertheless, with quality increased, we need higher bandwidth for trade-off. However, in most portable devices like cellular phone and PDA, the bandwidth is limited. Therefore, to attain the high quality video with less bandwidth, there were many compression standards, such as MPEG-1, MPEG-2, MPEG-4, H.261, and H.263, being developed for effective video transmission and storage. To further increase the compression efficiency, the Joint Video Team (JVT), which is organized by ISO MPEG and ITU-T VCEG, has finalize a new video coding standard H.264/AVC in May 2003 [1]. Experimental studies have shown that H.264/AVC can achieve about $50 \%$ bit rate saving with the similar perceptual video quality compare to other existing standards.

This research was partially supported by National Science Council under Contract \#NSC93-2213-E-006-011, Taiwan
H.264/AVC utilizes many advance features such as $4 \mathrm{x} 4$ integer transform, spatial intra-prediction in I-frame coding, $1 / 4$ pixel motion compensation, multiple reference frames and multiple block size for P-frame coding, context-based adaptive variable length coding (CAVLC) and context-based adaptive binary arithmetic coding (CABAC) for entropy coding, etc [2]. These features help H.264/AVC to achieve significantly high compression efficiency. However, these features also increase the complexity in H.264/AVC. Take variable block size motion estimation for example, there are totally seven block sizes, 16x16, 16x8, 8x16, 8x8, 8x4, 4x8, and $4 \times 4$. A macroblock can be encoded using one $16 \times 16$ block, two $16 \times 8$ blocks, two $8 \times 16$ blocks or four $8 \times 8$ blocks as shown in Fig. 1(a). Furthermore, one $8 \times 8$ block can be divided into two $8 \times 4$ blocks, two $4 \times 8$ blocks, or four $4 \times 4$ blocks as shown in Fig. 1(b). To encode a macroblock, we have to test all the inter modes to find the best inter mode with the least RD cost, which is very time-consuming. To reduce the complexity of inter mode decision, there are many algorithms being developed to accelerate the encoding time with tolerant performance degradation [3], [4].

In this paper, we will develop a fast inter mode decision algorithm to choose the best block type for each macroblock. The paper is organized as follows. First, the inter mode decision in H.264/AVC will be briefly introduced in Section II. In Section III, we will develop a fast algorithm based on hierarchical homogeneous detection and cost analysis to select the best inter mode effectively. Experimental results are shown in Section IV. Finally, we conclude the paper in Section V.

\section{INTER MODE DECISION IN H.264/AVC}

As we mentioned above, there are totally seven different block sizes can be used in interframe motion estimation. To select the best inter mode, the reference software JM10.1 provided two approaches: (1) RD optimization (RDO) and (2) non-RD optimization. The former uses the true distortion and true bits of each inter mode to calculate the RD cost while the later just adopts the sum of absolute Hadamard-transformed differences (SATD) and estimated motion vector cost of each inter mode to calculate the RD cost. It is obvious that we can achieve the 
best coding efficiency by using the true RDO method. However, it is too time-consuming to implement in realtime applications. Hence, we will focus on non-RDO method, where motion estimation is performed for all possible block sizes to choose the best inter mode with the least RD cost. The procedure contains two main steps: (1) motion vector search of each inter mode, and (2) partition mode search. For each inter mode, motion estimation is first performed for each block size $(16 \times 16,16 \times 8,8 \times 16,8 \times 8,8 \times 4$, $4 \times 8)$ to find the best motion vector. The best motion vector $\left(v_{x}, v_{y}\right)$ for a block size $N_{1} \mathrm{x} N_{2}$ is decided by minimizing a cost function:

$J_{N_{1} x N_{2}}\left(v_{x}, v_{y}\right)=S A(T) D_{N_{1} x N_{2}}\left(v_{x}, v_{y}\right)+\lambda\left(Q_{p}\right) R\left(M V D_{N_{1} x N_{2}}\left(v_{x}, v_{y}\right)\right)$,

where the $S A D$ is used for integer pixel motion estimation and the $S A T D$ is used for sub-pixel motion estimation, are defined as follows:

$$
\begin{aligned}
& \operatorname{SAD}_{N_{1} x N_{2}}\left(v_{x}, v_{y}\right)=\sum_{i=1}^{N_{1}} \sum_{j=1}^{N_{2}}\left|\hat{f}\left(i+v_{x}, j+v_{y}\right)-f(i, j)\right|, \\
& \operatorname{SATD}_{N_{1} x N_{2}}\left(v_{x}, v_{y}\right)=\sum_{i=1}^{N_{1}} \sum_{j=1}^{N_{2}}|c(i, j)| .
\end{aligned}
$$

In (2), $\hat{f}\left(i+v_{x}, j+v_{y}\right)$ and $f(i, j)$ denote the $(i, j)^{\text {th }}$ element of the reference block $\hat{\boldsymbol{F}}$ and the current block $\boldsymbol{F}$, respectively. In $(3), c(i, j)$ is the $(i, j)^{\text {th }}$ element of $\boldsymbol{C}$, which is the Hadamard transform of difference of $\hat{\boldsymbol{F}}$ and $\boldsymbol{F}$ depicted as

$$
\boldsymbol{C}=\frac{\boldsymbol{T}_{\mathrm{H}}(\hat{\boldsymbol{F}}-\boldsymbol{F}) \boldsymbol{T}_{\mathrm{H}}^{\mathrm{T}}}{2},
$$

with

$$
\boldsymbol{T}_{\mathrm{H}}=\left[\begin{array}{cccc}
1 & 1 & 1 & 1 \\
1 & 1 & -1 & -1 \\
1 & -1 & -1 & 1 \\
1 & -1 & 1 & -1
\end{array}\right] .
$$

In (1), $\lambda\left(Q_{p}\right)$ is an approximated exponential function of the quantization parameter $Q_{p}$ and $R(M V D)$ represents the rate cost contributed by motion vector difference $(M V D)$, is expressed as:

$$
M V D=\left|v_{x}-\bar{v}_{x}\right|+\left|v_{y}-\bar{v}_{y}\right|,
$$

where $\left(\bar{v}_{x}, \bar{v}_{y}\right)$ is the predicted motion vector defined in the standard. After motion vector search, we can get the motion vector for each sub-block and its corresponding cost $J_{s b}$. The next step is to find the best partition mode with least cost. The cost is defined as follows:

$$
J_{\text {mode }}=\sum_{s b \in \text { macroblock }} J_{s b},
$$

where $J_{\text {mode }}$ denotes the cost of the partition mode and $J_{s b}$ is the cost of the sub-block within the macroblock.

From the above, we can see that the computation complexity for deciding the best inter mode is very high since the motion estimation is performed for all possible block sizes. Therefore, how to accelerate the coding process and maintain the coding performance simultaneously is a very important subject in H.264/AVC interframe coding. In the next section, we will develop a fast algorithm for inter mode decision.

\section{Hierarchical Homogeneous Mode DETECTION AND COST ANALYSIS}

\section{A. Homogeneous Detection}

In the original inter mode decision algorithm, we have to select the best inter mode from all possible inter modes. To avoid the exhaustive search, $\mathrm{Wu}$ et al. proposed a fast algorithm to early terminate the inter mode search process by using Sobel operator and a recommended threshold to detect homogeneous blocks [3]. The main concept of the algorithm is that homogeneous blocks in the picture would have similar motion and are very seldom split into smaller blocks. Although this concept is indeed very useful for accelerating the inter mode decision process, it is hard to deal with some sequences (ex. Mobile) which contain complex background because of fewer homogenous blocks. The detection of spatial homogeneous blocks always fairs to correctly detect the complex-but-still background as larger blocks. To handle this problem, we suggest that we should first utilize temporal homogeneous detection to further improve the coding process. For example, if we find that the current macroblock is not spatial homogeneous, we will then perform $16 \times 16$ motion estimation to get the residue block and decide if the macroblock is temporal homogeneous or not. If the block is temporal homogeneous, the inter mode will also be decided as $16 \times 16$ mode. In this paper, an $N \times N$ block is said to be temporal homogeneous if its residue block satisfies the following condition:

$$
\sum_{i=1}^{N / 2} \sum_{j=1}^{N / 2}\left|r_{k}(i, j)-M_{k}\right|<T h, \quad k=0,1,2,3 .
$$

In (8), $r_{k}(i, j)$ and $M_{k}$ represent the $(i, j)^{\text {th }}$ element and the mean individually of the $k^{\text {th }}$ sub-block $R_{k}$ of the residue block, which is shown in Fig. 2. Th is an under-determined threshold. Here, we use (8) for temporal homogeneous detection since it needs less computation than Sobel operator but can achieve the similar performance. To determine the threshold, Th defined in (8), we can use the similar concept like [5], which develops an algorithm for the early detection of all-zero blocks. By using its developed threshold and make the condition more strictly for avoiding performance degradation, we set the threshold is $Q_{\text {step }}$ for a $4 \times 4$ block where $Q_{\text {step }}$ is the quantization step in H.264/AVC. Hence, the formula (8) can be written as:

$$
\sum_{i=1}^{N / 2} \sum_{j=1}^{N / 2}\left|r_{k}(i, j)-M_{k}\right|<\frac{N^{2}}{64} Q_{\text {step }}, \quad k=0,1,2,3
$$

\section{B. Cost Analysis}

If the current macroblock is neither spatial homogeneous nor temporal homogeneous, we will then conduct $8 \times 8$ motion estimation to obtain the cost of $8 \times 8$ mode. If we can determine the best inter mode is $16 \times 16$ mode or not in advance by analyzing the costs of $16 \times 16$ 
mode and $8 \times 8$ mode, we can save more computation. To achieve this, we first assume that the cost of $16 \times 16$ mode is less than the cost of $8 \times 8$ mode, that is:

$$
\begin{aligned}
& J_{16 \times 16 \bmod e}<J_{8 x 8 \bmod e} \\
& \Rightarrow S A T D_{6 x 16 \text { mode }}+M V \cos t_{16 x 16 \text { mode }}<S A T D_{8 x 8 \text { mode }}+M V \cos t_{8 x 8 \text { mode }},
\end{aligned}
$$

where $S A T D_{\text {mode }}$ and $M V \cos t_{\text {mode }}$ denote the total SATD and motion vector costs of the corresponding inter mode, respectively. In the above condition, because $16 \times 16$ mode is better than $8 \times 8$ mode, it is reasonable to assume that the best mode should be selected from $16 \times 16$ mode, $16 \times 8$ mode, or $8 \times 16$ mode. However, if the following condition satisfies:

$$
S A T D_{6 x 16 \text { mode }}-S A T D_{8 x 8 \text { mode }}<M V \cos t_{16 x 8 \text { mode }}-M V \cos t_{16 x 16 \text { mode }} \text {. }
$$

We don't need to perform the $16 \times 8$ motion estimation to get the cost of mode $16 \times 8$. Since

$$
\begin{aligned}
& \text { SATD }{ }_{6 x 8 \text { mode }}>\text { SATD }_{8 x 8 \text { mode }} \\
& \Rightarrow \text { SATD }_{6 x 16 \text { mode }}-\text { SATD }_{6 x 8 \text { mode }}<S A T D_{6 x 16 \text { mode }}-\text { SATD }_{8 x 8 \text { mode }} \\
& \Rightarrow S A T D_{6 x 16 \text { mode }}-S A T D_{6 \times 8 \text { mode }}<M V \cos t_{16 \times 8 \text { mode }}-M V \cos t_{16 x 16 \text { mode }} \\
& \Rightarrow S A T D_{6 x 16 \text { mode }}+M V \cos t_{16 x 16 \text { mode }}<S A T D_{6 x 8 \text { mode }}+M V \cos t_{16 x 8 \text { mode }}
\end{aligned}
$$

The inequality (16) means that the cost of $16 \times 16$ mode is less than that of $16 \times 8$ mode. For $8 \times 16$ mode, the condition is similar to $16 \times 8$ mode which just replaces $M V \cos t_{16 \times 8 \text { mode }}$ by $M V \cos _{8 \times 16 \text { mode }}$ in (12). Thus, the remaining problem is how to estimate $M V \cos t_{16 \times 8 \text { mode }}$ or $M V \cos t_{8 \times 16 \text { mode }}$ from $M V \cos t_{16 \times 16 \text { mode }}$ and $M V \cos t_{8 \times 8 \text { mode }}$. It is obviously that $M V \cos t_{16 \times 8 \text { mode }}$ or $M V \cos t_{8 \times 16 \text { mode }}$ is between $M V \cos t_{16 \times 16 \text { mode }}$ and $M V \cos t_{8 \times 8 \bmod e}$, and we can approximate them to the average of $M V \cos t_{16 \times 16 \bmod e}$ and $M V \cos t_{8 \times 8 \text { mode }}$. Therefore, the formula (12) can be rewritten as:

$$
S A T D_{6 x 16 \operatorname{mode}}-S A T D_{8 x 8 \text { mode }}<\frac{M V \cos t_{8 x 8 \text { mode }}-M V \cos t_{16 x 16 \text { mode }}}{2}
$$

By using (17), we can choose $16 \times 16$ mode as the best inter mode without performing $8 \times 16$ or $16 \times 8$ motion estimation. If (17) is not achieved, we then perform motion estimation for $8 \times 16$ and $16 \times 8$ modes and determine the final mode.

If the cost of $16 \times 16$ mode is larger than that of $8 \times 8$ mode, we then search the best $8 \times 8$ block subtype for each $8 \times 8$ block in the macroblock to get the candidate inter mode. The process from $8 \times 8$ mode to $4 \times 4$ mode is similar to the above process where each $8 \times 8$ block includes spatial homogeneous detection, temporal homogeneous detection, and cost analysis. Finally, the best inter mode is decided by selecting the inter mode with least cost from the candidate inter mode, $16 \times 8$ mode, and 8x16 mode.

\section{EXPERIMENTAL RESUlTS}

To evaluate our proposed algorithm, we test three sequences, Foreman, Mobile, and Bus with CIF format $(352 \times 288)$. And the experimental conditions are as follows: (1) the sequence type is IPPP...; (2) number of reference frame is 1 ; (3) search range is $33 \times 33$; (4) search algorithm is full search; (5) entropy coding method is CAVLC; (6) RD optimization (RDO) is disabled; (7) Hadamard transform is used; and (8) no random intra macroblock refresh in $\mathrm{P}$ pictures; and (9) the frame rate is 30 fps. Fig. 3 shows the RD curves achieved by the original method, only spatial homogeneous detection method (OSHDM), and the proposed method in different sequences. For the OSHDM, the threshold for homogeneous detection is set to 20000 for $16 \times 16$ blocks and 5000 for $8 \times 8$ blocks, which is recommend by [3]. For the proposed method, the threshold is set to 5000 for $16 \times 16$ block and 1250 for $8 \times 8$ block to lessen the PSNR degradation. From Fig. 3, we can see that the three curves are almost the same in three sequences which means our proposed method does not introduce any noticeable PSNR loss compare to the original method. Table I shows the time saving of motion estimation process of our proposed method and the OSHDM in different $Q_{p}$. From Table I, we can see that in sequences with complex scene (Mobile, Bus), the time saving of the OSHDM is not so much. Especially in Mobile, the time saving is just about $8 \sim 9 \%$ due to fewer homogeneous blocks. As for our proposed method, we can see that the time saving is still high even in complex sequences, which also demonstrates our proposed method is robust for various kinds of sequences. It is noticed that the time saving of our method is increase as $Q_{p}$ increase, which is owing to the temporal homogeneous detection by using formula (9). Table II shows the mode number per macroblock of the original method, the OSHDM, and the proposed method. The mode number per macroblock is defined as the equivalent $16 \times 16$ motion estimation times for each macroblock. Hence, for the original method, the mode_number/macroblock is always 7. From Table II, we can see the similar result and our proposed method can reduce nearly $50 \%$ mode computation for different kinds of sequences.

\section{Conclusions}

In this paper, we first introduced the process for inter mode decision in H.264 and then proposed a fast inter mode decision algorithm base on hierarhical homogeneous detection and cost analysis. Experimental results show that our proposed algorithm can deal with not only sequences with spatial homogeneous blocks (ex. Foreman) but also complex sequences (ex. Mobile, Bus). By using the proposed algorithm, we can save about 32 54\% coding time without introducing any noticeable performance degradation for various kinds of sequences, which is very helpful for H.264 interframe coding.

\section{REFERENCES}

[1] ITU-T Rec. H.264 / ISO/IEC 11496-10, "Advanced Video Coding", Final Committee Draft, Document JVTG050, March 2003.

[2] Thomas Wiegand, Gary J. Sullivan, Gisle Bjontegaard, and Ajay Luthra, "Overview of the H.264 / AVC Video Coding Standard," in IEEE Transactions on Circuits and Systems for Video Technology, July 2003. 
[3] D. Wu, F. Pan, K. P. Lim, S. Wu, Z. G. Li, X. Lin, S. Rahardja, and C. C. K, Fast Intermode Decsion in H.264/AVC Video Coding," in IEEE Transactions on Circuits and Systems for Video Technology, July 2005.

[4] Christos Grecos and Ming Yuan Yang," Fast Inter Mode Prediction for P Slices in the H264 Video Coding Standad," in IEEE Transactions on Broadcasting, June 2005.

[5] Yong Ho Moon, Gyu Yeong Kim, and Jae Ho Kim "An Improved Early Detection Algorithm for AllZero Blocks in H.264 Video Encoding," in IEEE Transactions on Circuits and Systems for Video Technology, August 2005.

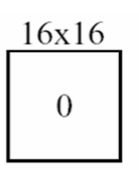

$8 \times 8$

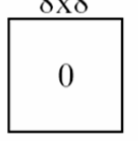

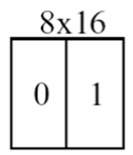

(a) Macroblock types
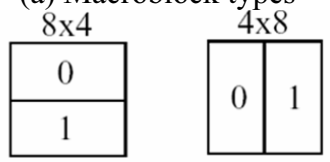

(b) $8 \times 8$ types

Fig 1. (a) Macroblock types (b) $8 \times 8$ types

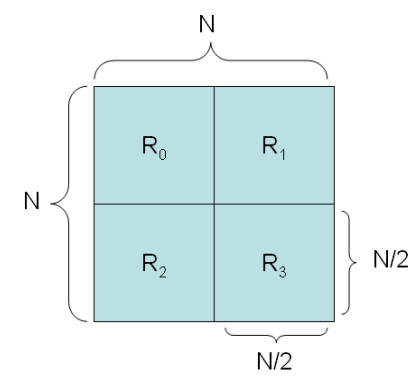

Fig 2. A Residue block with its sub-blocks.

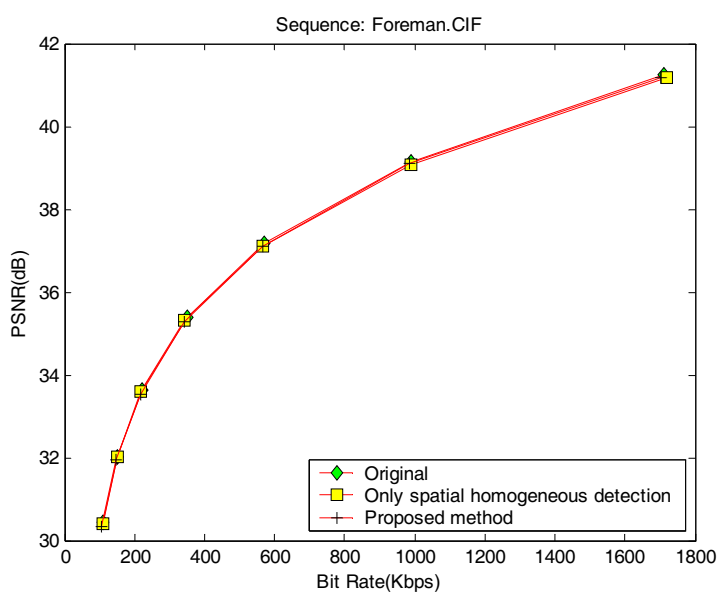

(a) Foreman

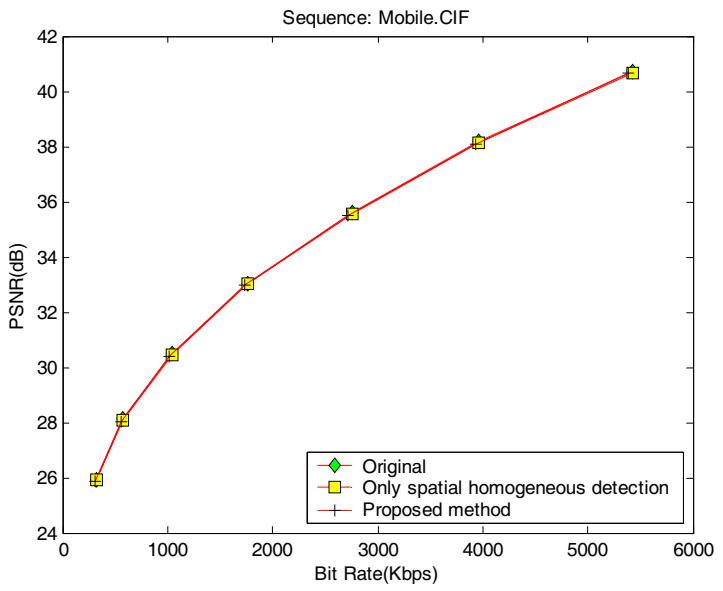

(b) Mobile

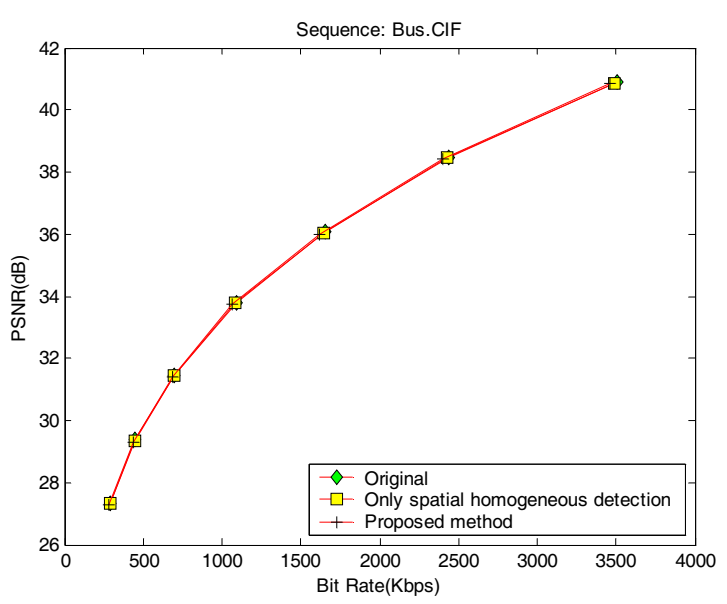

(c) Bus

Fig 3. RD curve of sequence (a) Foreman (b) Mobile (c)Bus

Table I. Time-saving (ME) in different Qp

\begin{tabular}{|c|c|c|c|c|c|c|}
\hline \multirow{2}{*}{ Sequence } & \multicolumn{2}{|c|}{ Foremat } & \multicolumn{2}{c|}{ Mobile } & \multicolumn{2}{c|}{ Bus } \\
\cline { 2 - 7 } & OSHDM & Froposed & OSHDM & Fropased & OSHDM & Froposed \\
\hline 26 & $39 \%$ & $40 \%$ & $9 \%$ & $33 \%$ & $19 \%$ & $32 \%$ \\
\hline 32 & $32 \%$ & $46 \%$ & $8 \%$ & $38 \%$ & $17 \%$ & $40 \%$ \\
\hline 38 & $30 \%$ & $54 \%$ & $9 \%$ & $45 \%$ & $12 \%$ & $44 \%$ \\
\hline
\end{tabular}

Table II. Mode_number/macroblock in different Qp

\begin{tabular}{|c|c|c|c|c|c|c|c|c|c|}
\hline \multirow{2}{*}{ Sequence } & \multicolumn{3}{|c|}{ Foreman } & \multicolumn{3}{c|}{ Mobile } & \multicolumn{3}{c|}{ Bus } \\
\cline { 2 - 11 } & Original & OSHDM & Froposed & Original & OSHDM & Froposed & Original & OSHDM & Froposed \\
\hline 26 & 7 & 4.11 & 3.51 & 7 & 6.07 & 4.21 & 7 & 5.55 & 4.10 \\
\hline 32 & 7 & 4.11 & 2.92 & 7 & 6.07 & 3.82 & 7 & 5.55 & 3.53 \\
\hline 38 & 7 & 4.11 & 2.31 & 7 & 6.07 & 3.28 & 7 & 5.55 & 3.02 \\
\hline
\end{tabular}

